\title{
Smart charging of electric vehicles with photovoltaic power and vehicle-to-grid technology in a microgrid; a case study
}

\author{
Mart van der Kam*, Wilfried van Sark \\ Utrecht University, Copernicus Institute of Sustainable Development, Heidelberglaan 2, 3584 CS Utrecht, The Netherlands
}

\section{H I G H L I G H T S}

- Simulation of microgrid including solar panels, electric vehicles and load demand.

- Comparison of smart EV charging control algorithms.

- Analysis of impact smart charging and V2G on PV self-consumption and peak reduction.

- Analysis of impact control algorithms on EV battery lifetime.

- Analysis of impact different microgrid configurations.

\section{A R T I C L E I N F O}

\section{Article history:}

Received 23 December 2014

Received in revised form 21 April 2015

Accepted 22 April 2015

Available online 15 May 2015

\section{Keywords:}

Smart grid

Photovoltaic electricity

Self-consumption

Electric vehicle

Vehicle-to-grid

\begin{abstract}
A B S T R A C T
We present a model developed to study the increase of self-consumption of photovoltaic (PV) power by smart charging of electric vehicles (EVs) and vehicle-to-grid (V2G) technology. Whereas previous studies mostly use large EV fleets in their models, our focus is on a smaller scale. We apply the model to a microgrid in Lombok, a residential neighbourhood in the city of Utrecht, the Netherlands. The microgrid consists of a $31 \mathrm{kWp}$ PV installation, an office, internet servers, three households, and two EVs. Three control algorithms are presented which manage the charging profile of multiple EVs either in real-time or using linear optimisation with predictions for PV power and electricity demand. We perform one-year simulations using data for PV power, EV use, and electricity demand. Simulations results are evaluated on PV self-consumption and peak demand reduction. In addition, we make qualitative statements on battery degradation resulting from the charging strategies based on several indicators. We also simulate changes in microgrid composition, for example by including more EVs. In the simulations, self-consumption increases from $49 \%$ to $62-87 \%$ and demand peaks decrease by $27-67 \%$. These results clearly demonstrate the benefits of smart charging EVs with PV power. Furthermore, our results give insight into the effect of different charging strategies and microgrid compositions.
\end{abstract}

(c) 2015 Elsevier Ltd. All rights reserved.

\section{Introduction}

The transition to low carbon energy and transport systems requires not only the large-scale adoption of clean technologies and efficiency measures, but also new energy management strategies to efficiently incorporate these innovations in the existing infrastructure. Issues related to the grid integration of clean technologies can occur both at the energy supply side, with technologies such as photovoltaics (PV), and on the demand side, with technologies such as electric vehicles (EV). Sophisticated energy management can help solving these issues and optimise allocation

\footnotetext{
* Corresponding author. Tel.: +31 302537534.

E-mail address: M.J.vanderKam@uu.nl (M. van der Kam).
}

of resources, for instance by charging EVs with PV power instead of electricity from coal or gas-fired power plants.

In the residential sector, there is an imbalance between PV power supply and electricity demand. PV installations produce most electricity during the day [1,2], while electricity demand of households peaks in the morning and evening. Furthermore, typical EV charging patterns contribute to existing peaks in household electricity demand ${ }^{1}$. A higher penetration level of PV and EVs will increase power transport over the electricity grid, requiring grid investments to prevent overloads [3,4]. Several countries in Europe have started implementing policies to stimulate the

\footnotetext{
E-laad [Internet]. Opladen elektrische auto's zorgt voor piekbelastingen [update 2013 May 6th; cited 2014 May 14th] Available from: <http://www.e-laad.nl/nieuws opladen-elektrische-autos-zorgt-voor-piekbelastingen-2/>. In Dutch.
} 
self-consumption of locally generated energy [5]. Self-consumption of PV power should increase to ensure grid stability and functioning.

In a smart grid the traditional electricity grid or microgrid (i.e. a local, low-voltage distribution system) is combined with information and communication technologies [6]. Load shifting is an essential aspect of smart grids and can be used to increase self-consumption of PV power [7] and off-peak charging of EVs [8]. An important advantage of EVs in smart grids is that they can be used both as a flexible demand source and as a storage option, using vehicle-to-grid (V2G) technology [9-13].

In this paper we use a case study to model and simulate the application of smart charging algorithms for EVs. Simulation studies on using EVs for integration of PV in the grid mostly use a high level of aggregation of EVs in their models. For example, two studies have been found that consider the case of using parking lots to integrate EV and PV. Tulpule et al. [14] have performed a study for a parking lot at a workplace in Columbus, OH, USA and Los Angeles, CA, USA and show the feasibility of such a system as compared to home charging both in terms of costs and $\mathrm{CO}_{2}$ emissions. Birnie [15] considered a parking lot in New Jersey, NJ, USA and used a simple approach to determine that most driving needs could be met by solar power in the summer, but not in the winter. Other studies consider EV fleets at a city or region level. For instance, Zhang et al. [16] show that by using smart charging one million EVs combined with one million heat pumps can reduce excess PV power by $3 \mathrm{TW} h$ for the Kansai Area, Japan. Drude et al. [17] study PV and V2G strategies in urban regions in Brazil. They conclude the EVs can be used for grid-stabilisation, but that adequate energy policies are needed to avoid destabilisation due to too many cars offering storage for V2G. Tuffner et al. [18] simulated a distribution system (IEEE 123-node) for Phoenix, AZ, USA weather conditions. They conclude that penetration rates of EV and PV have to be high $(>50 \%)$ to have a significant impact on the network but that the synergy of these technologies has significant benefits for these high penetration rates.

According to Guille and Gross [19], EV batteries are too small to make a significant impact on the grid by themselves. However, large-scale deployment of V2G faces many socio-technical barriers [20]. Our study aims to show the benefits of using EVs and smart grid technology in a microgrid, since such a small-scale project can be realised in the near future. These innovative pilot projects are pivotal in realising the transformation of socio-technical systems such as the energy system as they allow the small-scale experimentation with alternatives to the current system [21-23]. Furthermore, studying this project allows us to combine specific real-world empirical data on PV power supply, load demand and EV use. This paper thus contributes to the existing literature by exploring alternatives to large-scale deployment of using EVs for integration of PV in the electricity grid.

Our case study is LomboXnet ${ }^{2}$, a company providing internet connection to about 2500 people in Lombok, a neighbourhood in Utrecht, the Netherlands. LomboXnet has the ambition to run its activities on locally produced solar power and provides PV power to three houses in the neighbourhood. The company has two battery EVs, which are used for car sharing. Car sharing is becoming increasingly popular worldwide [24] and also in the Netherlands ${ }^{3}$ and it has a great potential to reduce the environmental impact of personal transportation [25-27]. When used for car sharing, the EVs are regularly stationed at the charging station, making them suitable for grid balancing. This in contrast to other types of EV use such as commuting. The combination of PV, EV, smart grid and car sharing makes

\footnotetext{
2 Company website available from: <http://www.lombox.nl>. In Dutch.

${ }^{3}$ KVPP [Internet]. Snelle opkomst onderling autodelen [update 2013 June 20th; cited 2014 September 5th] Available from: <http://kpvvdashboard-4.blogspot.nl/ 2013/06/snelle-opkomst-onderling-autodelen.html>. In Dutch.
}

LomboXnet an excellent case for studying the integration of clean technologies.

Our research objective is to determine the potential for increasing the self-consumption of PV power with smart charging of EVs for LomboXnet. We simulate three different charging algorithms. The first algorithm uses real-time information, the second uses real-time information and V2G, and the third is an optimisation algorithm using predictions for PV power supply and load demand and V2G.

The remainder of this paper is organised as follows. In Section 2 we introduce our model. Section 3 presents our control algorithms and Section 4 the indicators used. Section 5 contains simulation results. In Section 6 we discuss our method and results and in Section 7 we draw our final conclusions.

\section{Model description}

In this section we present the structure and components of our model. Fig. 1 presents an overview of the microgrid of LomboXnet. The five main components of the microgrid are the PV installations, the energy management system, the uncontrollable load, the controllable load, and the connection to the main grid. The uncontrollable load consists of the office building, the internet servers and three households, each with a distinctive type of load curve. The demand from the office building peaks during the day, the internet servers have a constant demand and household demand peaks during the morning and evening. The PV power is used to cover both the uncontrollable and the controllable load. In case of PV power shortage, electricity is drawn from the main grid. In case of excess PV power, electricity is fed back into the main grid.

\section{1. $P V$}

The PV installations provide electricity to the microgrid. In total, $31 \mathrm{kWp}$ is installed with a solar energy yield of about $25 \mathrm{MW}$ her year and a performance ratio (PR) of $74 \%$ as measured for the year 2013. The PR is a measure for the overall losses of a PV system and is defined as the ratio of final energy yield of the PV system in $\mathrm{kW} \mathrm{h/kWp} \mathrm{to} \mathrm{a} \mathrm{reference} \mathrm{yield,} \mathrm{which} \mathrm{takes} \mathrm{only} \mathrm{solar} \mathrm{irradiation}$ into account [2]. In the Netherlands, the average PR is 78\% [28]. The below average performance of the LomboXnet PV system is explained by the partial shading of several solar panels during the day. The PV power output is directly measured at the solar inverter and available with a resolution of an hour.

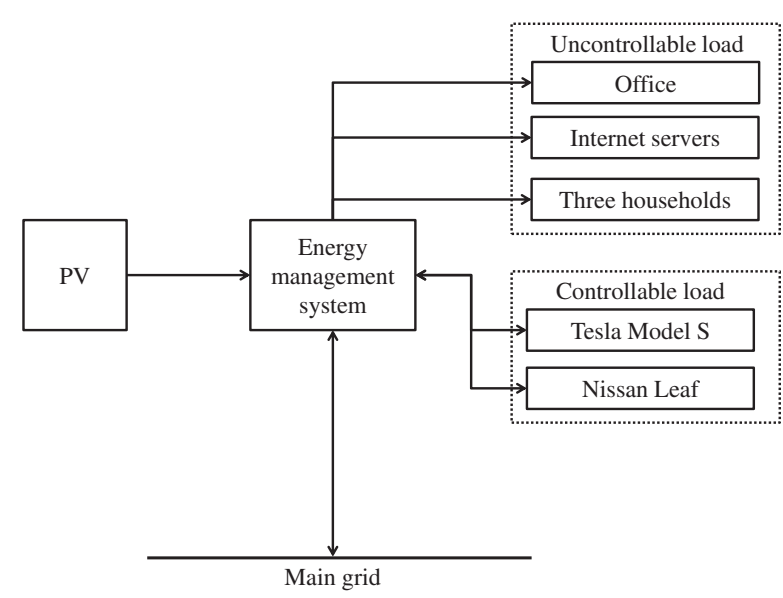

Fig. 1. Microgrid at LomboXnet, arrows indicate power flows. 


\subsection{Uncontrollable load}

The uncontrollable load is the part of the total load that cannot be controlled by the energy management system and must be met at all times. It consists of the electricity demand for the office, the LomboXnet servers and the three connected households. Electricity is provided from the PV installations (first priority), the EV batteries (second priority, when V2G is available) and the main grid.

The load demand of the office and internet servers is measured at LomboXnet with an hourly resolution. The yearly demand in 2012 was $27 \mathrm{MW} \mathrm{h}$. The majority of this demand (19 MW h) is from the internet servers, which constantly use around $2.2 \mathrm{~kW}$. Because there are no measurements available for the households, we use an estimate for the demand. The demand profiles are estimated using a data-set containing 400 unique household profiles as provided by Claessen [29]. The data-set is based on measurements from Liander, the largest utility company in the Netherlands. We select households with a yearly average electricity demand within $30 \%$ of $3680 \mathrm{~kW} \mathrm{~h}$, representative for the houses in the microgrid, resulting in a total of 153 households. This average is higher than the Dutch yearly average of $3480 \mathrm{~kW} \mathrm{~h} \mathrm{[30].}$ Fig. 2 presents an Example 24-h load profile. In the figure the peaks at different times caused by the different loads are clearly visible.

\subsection{Electric vehicles}

Two battery EVs are currently available: a Tesla Model S and a Nissan Leaf. The technical specifications are presented in Table 1. Furthermore, a minimum energy level of $20 \%$ of the battery capacity is assumed, as well as a power conversion efficiency of $90 \%$.

The EVs are used for car sharing. Experience at LomboXnet has shown that each car is used for three trips per week on average. These trips have a duration of $3-6 \mathrm{~h}$, a minimum distance of $20 \mathrm{~km}$, and a maximum distance of the full EV range. These numbers are used to simulate driving patterns via a pseudorandom
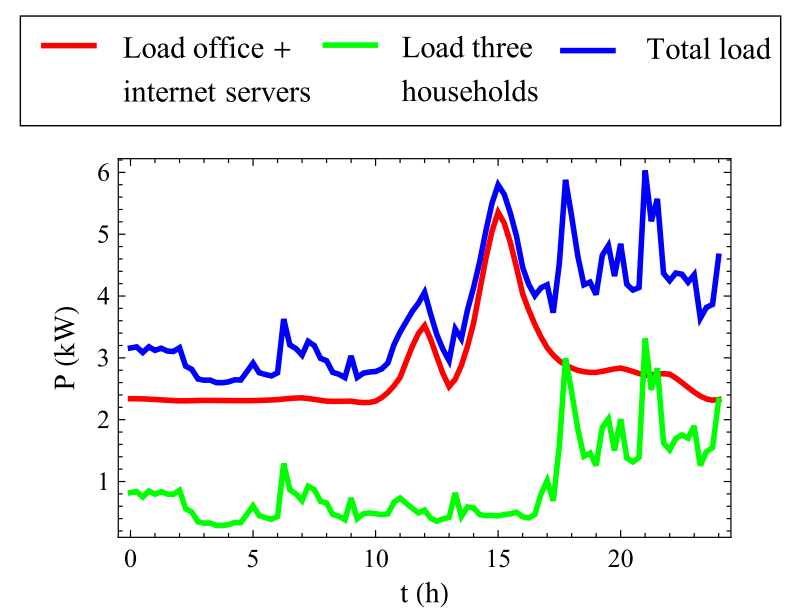

Fig. 2. Load profile example.

Table 1

Technical specifications of EVs. Data source: U.S. Environmental Protection Agency [update 2014 July 25th, cited 2014 July 28th] available from: http:// www.fueleconomy.gov.

\begin{tabular}{|c|c|c|}
\hline & Tesla Model S & Nissan Leaf \\
\hline Battery capacity (kW h) & 85 & 24 \\
\hline Energy consumption ( $\mathrm{kWh} \mathrm{h} / \mathrm{km})$ & 0.233 & 0.211 \\
\hline Range $(\mathrm{km})$ & 340 & 150 \\
\hline Charging power (kW) & 22 & 6.6 \\
\hline
\end{tabular}

number generator that decides (a) if a trip is made that day, with a $3 / 7$ chance of a trip taking place, (b) trip duration, between the minimum and maximum value, and (c) trip distance, between the minimum and maximum value. For our purposes, a trip means that the EVs are not available at the charging stations and that a certain amount of energy is consumed for driving. The electric vehicles need around $10 \mathrm{MW}$ her year in total to make the trips.

\subsection{Changes in microgrid composition}

LomboXnet considers several extensions of the microgrid, which we also simulate. Possible extensions in the foreseeable future include extra solar panels ( $3 \mathrm{kWp}$ ), two extra households, and three extra EVs. The extra EVs are all Nissan Leafs and include one EV used similar to the other Nissan Leaf and two private EVs, used for commuting. The latter EVs make a trip every workday between 8:00 and 19:00 with a duration of 6-10 h and a distance of $60-90 \mathrm{~km}$.

Furthermore, we run simulations for certain changes in microgrid composition. We vary EV model, average electricity demand of the households, and the number of trips the EVs make per week.

\section{Control algorithms}

In this section we present our three simulated control algorithms. Two are based on real-time (RT), with and without the V2G option, and one is based on linear programming (LP). All algorithms are based on a centralised approach: the energy management system decides the EV charging patterns, not the individual EVs. We use these three charging schemes to evaluate system performance with RT versus planning strategies and to see the effect of using V2G. The algorithms decide the charging patterns of the EVs, using them as a flexible demand source and in the case of V2G as an electricity storage device. The goal of using such a system for LomboXnet is to increase the consumption of PV power within the microgrid. Our algorithms do not incorporate other factors that might be of interest in addition to PV self-consumption, such as electricity price and power quality. However, the algorithms are easy to program and suitable for our purpose: demonstrating the potential role of EVs in this microgrid.

With our first RT algorithm, RT Control, the EVs only use PV power to charge the batteries, unless there is more demand than $\mathrm{PV}$ power to make a trip. In this algorithm V2G is not available. There are technological as well as social barriers to V2G technology [20], so it is interesting to explore strategies without V2G. Our second RT algorithm, RT Control + V2G, has the V2G option is available. The EV charges with PV power as much as possible and discharges energy when not enough PV power is available for the uncontrollable load.

While RT Control +V2G is expected to increase the PV self-consumption it is not necessarily the optimal strategy for EV charging. The algorithm only uses real-time information and is therefore not able to optimise the charging pattern for a longer time period. This is why we also introduce an optimisation algorithm. Constrained optimisation is a technique used often in research on applications of smart grids, recent examples include [31-34]. We use linear programming, because increasing PV self-consumption can be formulated as a linear optimisation problem. Furthermore, we have previously shown that LP can reduce peaks significantly more than RT algorithms [35]. For LP, PV power supply and load demand must be known in advance, so predictions are necessary. Therefore, we evaluate our algorithm in two ways, with and without taking uncertainties in predictions into account. 


\subsection{No Control (reference charging scheme)}

Without a smart grid program, the EVs, if connected, will always charge at maximum capacity until they are full. This reference charging scheme is called No Control and is represented by Eq. (1).

$P_{i}(t)= \begin{cases}P_{i}^{\max } & \text { if } E_{i}(t-1)<C_{i} \text { and } t \in t_{i}^{l} \\ 0 & \text { else }\end{cases}$

With $i$ the EV index, $P_{i}(t)$ the EV charging power at time step $t, P_{i}^{\max }$ the maximum charging power, $E_{i}(t)$ the energy contained in the battery at time step $t, C_{i}$ the battery capacity, and $t_{i}^{l}$ the time steps for which the EV is at the loading station.

In this reference charging scheme, the charging patterns of the EVs cannot be controlled. We model the EV charging patterns as controllable and compare these results to the reference simulations.

\subsection{Real-time control algorithms}

In Fig. 3 we show the flow of information for the RT algorithms. The EV charging patterns are decided based on PV power, uncontrollable load, planned EV trips and the state of charge (SOC) of the EV batteries. The SOC is determined based on how much energy the EV charges, discharges, and uses for a trip.

The RT algorithms use the difference between PV power supply and load demand for every time step $t$. Taking into account the energy content of the EV the charging pattern is then decided. If there is more PV power than electricity demand, the EV starts charging using the excess PV power until the battery is full or until there is no more excess PV power. The EV extracts energy from the grid only when there is a shortage of PV power to make a trip. If there is insufficient PV power to cover load demand, energy can be extracted from the EV. Thus, the algorithms distinguish three power flows as represented in Eq. (2)

$P_{i}(t)=P_{i}^{P V}(t)+P_{i}^{\text {grid }}(t)-P_{i}^{\text {out }}(t)$

With $P_{i}^{P V}(t)$ the charging power from PV, $P_{i}^{g r i d}(t)$ the power drawn from the grid, $P_{i}^{\text {out }}(t)$ the discharged power. These three components cannot exceed the maximum charging power, see Eq. (3).

$\left|P_{i}(t)\right| \leqslant P_{i}^{\max }$

With $P_{i}^{\max }$ the maximum charging power. Note that it is assumed that the maximum discharging power equals the maximum charging power.

Taking into account the energy needed for trips and maximum charging power, the required amount of energy in an EV for each time step, $E_{i}^{\text {req }}(t)$ is defined in Eq. (4).

$E_{i}^{r e q}(t)= \begin{cases}E_{i}^{t \text { trip }}-P_{i}^{\text {max }} \cdot\left(t_{i}^{\text {trip }}-t\right)+E_{i}^{\text {min }} & \text { if } t \in\left(t_{i}^{\text {trip }}-\frac{E_{i}^{\text {trip }}}{P_{i}^{\text {mix }}}, t_{i}^{\text {trip }}\right) \\ E_{i}^{\text {min }} & \text { else }\end{cases}$

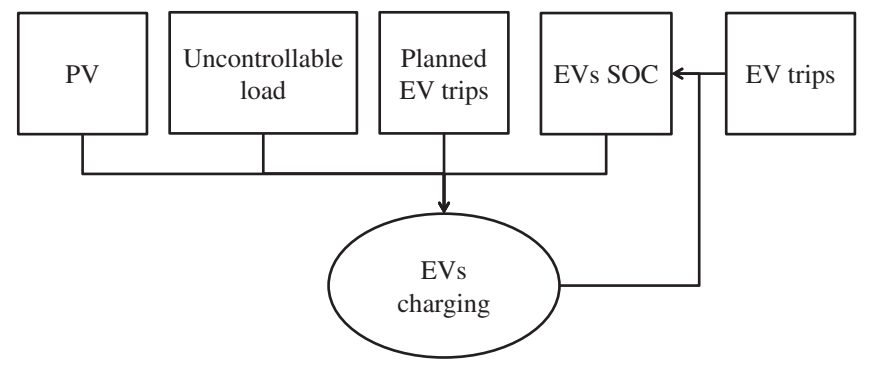

Fig. 3. Flow of information for RT Control algorithms.
With $t_{i}^{\text {trip }}$ the time of the next trip, $E_{i}^{\text {trip }}$ the energy required for the next trip and $E_{i}^{\min }$ the minimum energy in the battery. The 'if part of Eq. (4) describes that when a trip is planned, the energy level at the start time of the trips must be the energy needed for the trip plus the minimum energy level. The energy level of the time steps before the start time of the trip also have to be at a certain level, taking into account the energy required for the trip, the maximum charging power and the time left to complete charging. At all other time steps the required energy in the EV is the minimum energy level of the EV, as the described by the 'else' part of Eq. (4).

When using multiple EVs, a priority function $f_{i}$ is needed. First, an urgency value $U_{i}$ is assigned to each vehicle. $U_{i}$ is based on how much time it takes to charge the vehicle to a sufficient energy level for the next trip and the time left to achieve this, see Eq. (5). $f_{i}$ is then calculated as $U_{i}$ proportional to the sum of $U_{i}$ for all vehicles, see Eq. (6).

$\begin{aligned} U_{i}(t) & =\left(\frac{t_{i}^{\text {trip }}-t}{t_{i}^{\text {trip }}-t-\left(E_{i}^{\text {trip }}-E_{i}(t-1)\right) / P_{i}^{\max }}\right)^{u} \\ f_{i}(t) & =\frac{U_{i}(t)}{\sum_{i} U_{i}(t)}\end{aligned}$

Eq. (5) contains a power factor $u$; in the simulations $u=2$ was used so that the effect is increased for EVs that have a high urgency for charging compared to $u=1$. However, it was found that the value for $u$ has little effect on the outcome when evaluating system performance for $u=1,2$ or 3 , so final results should apply for all these values for $u$.

\subsubsection{RT Control}

With RT Control we model the real-time smart charging of the EVs. The EVs start charging when excess PV power is available, unless there is insufficient PV power to charge enough for a trip. The loading patterns are defined by Eqs. (7) and (8).

$P_{i}^{P V}(t)=\left\{\begin{array}{lll}\eta_{i} f_{i}(t)\left(P_{P V}(t)-P_{\text {load }}(t)\right) & \text { if } \quad \begin{array}{l}P_{\text {load }}(t)<P_{P V}(t) \\ \end{array} & \text { and } E_{i}(t-1)<C_{i} \\ & \text { and } t \in t_{i}^{l}\end{array}\right.$

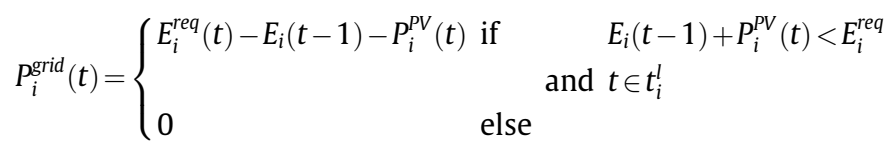

With $P_{P V}$ the PV power, $P_{\text {load }}$ load demand and $\eta_{i}$ the charging efficiency. If there is more PV power than electricity demand, the EV starts to charge until it is full or until there is no more excess PV power, see Eq. (7). Eq. (8) defines that the EV only extracts energy from the grid when there is shortage of PV power to make a trip.

\subsection{2. $R T$ Control + V2G}

With RT Control + V2G energy can be extracted from the EV to cover load demand. Eq. (9) is added to the equations of RT Control.

$$
P_{i>1}^{\text {out }}(t)=\left\{\begin{array}{lll}
\eta_{i}^{-1} \frac{1-f_{i}(t)}{N-1}\left(P_{\text {load }}(t)-P_{P V}(t)\right) & \text { if } & P_{\text {load }}(t)>P_{P V}(t) \\
& & \text { and } E_{i}(t-1)>E_{i}^{\text {req }}(t) \\
& \text { and } t \in t_{i}^{l}
\end{array}\right.
$$

With $N$ the number of EVs. Note that in the case of only one EV no priority function is needed and the factor $\frac{1-f_{i}(t)}{N-1}$ is set to 1 . 


\subsection{Linear programming}

Increasing self-consumption of PV power by controlling the charging pattern of an EV can be described as a linear optimisation problem and solved by using linear programming. Our objective is to maximise the use of PV, both for charging the EV and using energy from the EV to cover load demand, and to minimise energy drawn from the grid. This is done under constraints related to factors such as PV power, uncontrollable load and trips to be made. All constraints must be known in advance, therefore we have to use predictions for PV power and the uncontrollable load. We assume the linear program is run at midnight ( $t=0$ is midnight). Fig. 4 presents the information flows for the LP control algorithm. Note that in contrast to the RT algorithms there is no dynamic updating.

For the formulation of the problem, we use $P_{i}^{P V}(t), P_{i}^{\text {grid }}(t)$ and $P_{i}^{\text {out }}(t)$ for all time steps $t$ as variables. We formulate our objective function as follows:

$\sum_{i} \sum_{t} k\left(P_{i}^{P V}(t)+P_{i}^{\text {out }}(t)\right)-P_{i}^{\text {grid }}(t)$

With $k$ a factor to determine the importance of using PV power over drawing energy from the grid. We have based our value of $k$ on trial and error. $k$ must not be too low $(\sim 1)$, because then little solar energy is used, and not too high $\left(\sim 10^{3}\right)$, because then little energy is extracted from the EV battery. For our simulations, we have set $k=100$. Our final results are not sensitive to the exact value of $k$ in this order of magnitude. The objective function is maximised, subject to the following constraints:

$\left|P_{i}(t)\right| \leqslant P_{i}^{\max }$

$\sum_{t^{\prime}=1}^{t}\left(\eta_{i}\left(P_{i}^{P V}\left(t^{\prime}\right)+P_{i}^{\text {grid }}\left(t^{\prime}\right)\right)-\eta_{i}^{-1} P_{i}^{\text {out }}\right) \leqslant C_{i}+E_{i}^{\text {trip }}(t)-E_{i}(0), \forall t, i$

$\sum_{t^{\prime}=1}^{t}\left(\eta_{i}\left(P_{i}^{P V}\left(t^{\prime}\right)+P_{i}^{\text {grid }}\left(t^{\prime}\right)\right)-\eta_{i}^{-1} P_{i}^{\text {out }}\right) \geqslant E_{i}^{\text {trip }}(t)-E_{i}(0), \forall t, i$

$\sum_{i} P_{i}^{P V}(t) \leqslant \max \left[P_{P V}(t)-P_{\text {load }}(t), 0\right], \forall t$

$\sum_{i} P_{i}^{\text {out }}(t) \leqslant \max \left[P_{\text {load }}(t)-P_{P V}(t), 0\right], \forall t$

With dummy variable $t^{\prime}$. Note that in this case, $P_{i}^{\max }(t)$ is represented as a function of $t$, contrary to earlier in this paper. This is done to include that $P_{i}^{\max }$ is in fact zero when an EV is not at the loading station.

Constraint (11) ensures that the maximum charging power is not exceeded. Constraints (12) and (13) ensure that the energy in the EV does not exceed the battery capacity and is sufficient for trips. Constraints (14) and (15) ensure that not more solar energy is charged than the excess of PV power or discharged than the shortage of PV power. Furthermore, all variables are non-negative.

Executing the linear program requires all variables of the constraints to be known in advance. Therefore, we evaluate the

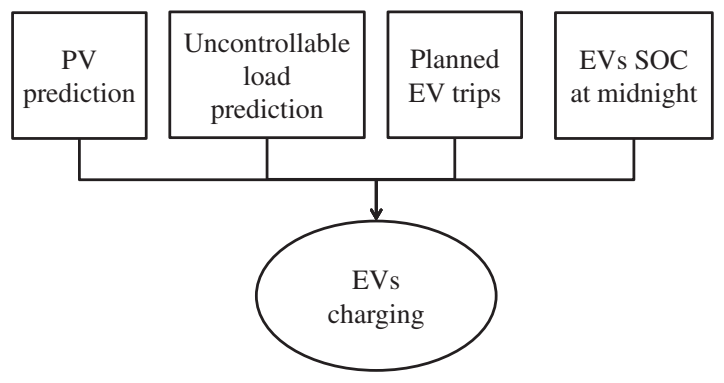

Fig. 4. Flow of information for LP control algorithm. algorithm in two ways, with perfect information, LP - Perfect Information, and with simulated uncertainties in predictions, LP - Uncertainties. Load demand predictions are based on the pattern from the previous day. An exception is made for weekends, since weekend load demand differs significantly from weekdays. However, the data we use includes only a week per household. Because of this limitation predictions for Saturdays will be based on data for Sundays and predictions for Mondays will be based on data for Tuesdays. This results in the following equations:

For Tuesdays, Wednesdays, Thursdays, Fridays and Sundays:

$P_{\text {load }}^{\text {prediction }}(t)=P_{\text {load }}^{\text {real }}(t-24 \mathrm{~h})$

For Mondays and Saturdays:

$P_{\text {load }}^{\text {prediction }}(t)=P_{\text {load }}^{\text {real }}(t+24 \mathrm{~h})$

The input for PV is based on PV power predictions. It is assumed that prediction deviates from the real value with standard deviation $\sigma$, as follows:

$P_{P V}^{\text {prediction }}(t)=P_{P V}^{\text {real }}(t) \pm \sigma$

While it is possible for $P_{P V}$ to be zero, there is a maximum PV yield per time step. To take this into account for each month a profile of maximum PV power $P_{P V}^{\max }$ is created. This is done by fitting the function defined in Eq. (19) to the maximum yield found in the datasets for each month.

$P_{P V}^{\max }(t)=a \cdot \exp \left(-b^{2}\left(t-t_{\max }\right)^{2}\right)$

After fitting values for $a$ (maximum yield), $b$ (spread) and $t_{\max }$ (time step of maximum PV power) $P_{P V}^{\max }$ for each month is defined. $P_{P V}^{\text {real }}(t)$ can never exceed $P_{P V}^{\max }(t)$, no matter how big $\sigma$ is. In our simulations we assume $\sigma$ to be $10 \%$. We have tested the results for changes in $\sigma$ and found that as long as $\sigma$ is below a threshold value around 20\% changes in $\sigma$ do not significantly affect results. For LP Uncertainties the algorithm is executed with the predicted values, while it is evaluated with the real values.

We use linear programming because we want to model an optimisation method for increasing self-consumption. However, some practical issues for our simulations arise by including linear programming. First of all, we do not include the effect that EVs will charge significantly slower when the SOC of a battery approaches $\mathrm{SOC}=1$. This mechanism cannot be included in the way the linear programming algorithm is defined, because it would alter the constraints for each variation of the variables. For a fair comparison of control algorithms it was therefore chosen not to include this effect in any of the simulations. Furthermore, solving the linear program takes significantly more time than running simulations for the RT algorithms. For this research not enough time or computer power was available to run simulate more than $24 \mathrm{~h}$ for linear programming. This is an issue because for every simulation a random value for the SOC at $t=0$ is set, significantly effecting results as opposed to simulating longer time periods. To provide an estimation of this effect we perform a sensitivity analysis for the value of SOC at $t=0$.

\section{Indicators}

In this section we present our indicators. Section 4.1 presents our system performance indicators. Furthermore, we use indicators for battery degradation, since the feasibility of a V2G system is dependent on the impact it has on EV battery lifetime. We discuss this in Section 4.2 . 


\subsection{System performance}

We evaluate system performance on self-consumption (SC), energy sent to the grid and relative peak reduction (RPR). Based on the simulations the potential for increasing self-consumption is calculated. Self-consumption is defined as the relative amount of PV power used by the households and the EVs, see Eq. (20).

$\mathrm{SC}(T)=\frac{\sum_{t=T 0}^{T} \min \left[P_{P V}(t), P_{\text {load }}(t)+\sum_{i} P_{i}(t)\right]}{\sum_{t=T 0}^{T} P_{P V}(t)} \cdot 100 \%$

With $T$ the period that is evaluated and $T_{0}$ the start time of period $T$.

As an absolute indicator we give the amount of energy sent to the grid, as defined in Eq. (21):

$E_{\text {grid }}^{\text {in }}(T)=\sum_{t=T_{0}}^{T} \max \left[P_{P V}(t)-P_{\text {load }}(t)-\sum_{i} P_{i}(t), 0\right]$

Finally we use relative peak reduction for evaluation. RPR compares the deviation of the average of the load demand for the main grid $P_{\text {grid }}^{\text {tot }}(t)$, defined in Eq. (22), with a control algorithm, $P_{\text {grid,control }}^{\text {tot }}(t)$, to the No Control reference scenario, $P_{\text {grid,nocontrol }}^{\text {tot }}(t)$, and is defined in Eq. (23).

$$
\begin{aligned}
& P_{\text {grid }}^{\text {tot }}(t)=P_{\text {load }}(t)-P_{P V}(t)+\sum_{i} P_{i}(t) \\
& \operatorname{RPR}(T)=\left(1-\frac{\sum_{t=T 0}^{T} \mid P_{\text {grid,control }}^{\text {tot }}(t)-\overline{P_{\text {grid,control }}^{\text {tot }} \mid}}{\sum_{t=T 0}^{T} \mid P_{\text {grid,nocontrol }}^{\text {tot }}(t)-\overline{P_{\text {grid,nocontrol }}^{\text {tot }} \mid}}\right) \cdot 100 \%
\end{aligned}
$$

\subsection{Battery degradation}

Factors that impact battery lifetime are: number of cycles; operation temperature; rates of charge and discharge; depth of discharge (DOD), SOC and total energy withdrawn [36]. Battery lifetime is often expressed as cycle-lifetime. Manufacturers commonly provide information on cycle-lifetime as a function of DOD for a battery that is discharged from a SOC of $100 \%$. This data is not suitable for our purposes since the charging pattern of the EVs in the simulations consists for a large part of multiple, smaller cycles which do not start at a SOC of $100 \%$. Several models have been proposed to quantify the impact of V2G on battery lifetime [36-38]. Furthermore, several EV battery degradation models are available that take smaller battery cycles into account [39-42]. However, applying these models to our simulations will require making many assumptions regarding factors such as operation temperature and voltage, since the only output of our model is the charging pattern of the EVs. It is outside the scope of this paper to present a complete battery model. However, to give an indication of the impact of the control algorithms on battery lifetime we use three indicators: energy throughput, rate of charge and discharge and SOC. We exclude the battery degradation due to the driving cycles of the EVs, since the output of our simulations only contain information on the charging patterns when the EVs are at the charging station.

We define energy throughput as the total amount of energy charged and discharged in MW h per year. To evaluate the economics of V2G, Kempton and Tomić [37] express battery lifetime in energy throughput as a function of cycle lifetime, battery capacity and DOD for which the cycle lifetime was determined. The authors thus assume a linear relation between energy throughput and battery degradation rate at constant DOD. In an experimental study, Peterson et al. [43] found energy throughput is the strongest indicator for EV battery degradation, regardless of DOD, and found a linear relation between energy throughput and battery degradation rate. In a simulation study, Bishop et al. [36] found EV battery degradation to be most dependent on energy throughput and found a square root relation between energy throughput and battery degradation rate. The latter two studies thus agree that energy throughput is the most important factor in measuring battery degradation, but disagree on the particular relationship.

We present our results for SOC as yearly average. Cycling at high SOC values is found to have a negative effect on EV battery lifetime $[40,41]$. However, battery degradation can also result from overdischarge, so too low SOC values must also be avoided. In our simulations we use a minimum SOC of $20 \%$ to prevent overdischarge, similar as in [14], though some other studies use a minimum SOC of $30 \%[16,34,44]$.

For rates of charge and discharge we present our results as yearly average and as a frequency distribution. We take the yearly average of the absolute value of the charging rates, assuming charging and discharging have the same effect on battery lifetime. Higher charging rates result in higher battery degradation [36,4143].

\section{Results}

This section presents our simulation results. For every case we have run a one-year simulation with 15-min time steps. In Section 5.1 we show examples of simulation to illustrate the difference between the algorithms. In Section 5.2 we show our main results, Section 5.3 presents our results for the battery degradation indicators, and in Section 5.4 we show our results of changes in microgrid composition.

\subsection{Example simulations}

Fig. 5 presents examples of 24-h simulations. This figure illustrates the differences in EV charging strategies and how they affect self-consumption. The difference should be minimised between the net load and the PV power. The examples show a day in July, which represents high PV yield and low household load, since the effects of the control systems are more clear here then in a winter situation. In the examples both EVs are away on a trip; in the complete year-long simulations this will occur only $18 \%$ of the time. The EV batteries are half full at $t=0$ in all examples, except in the case of No Control, since the batteries have been fully charged the day before.

In the case of No Control (Fig. 5a), the EVs only charge after a trip: in the late afternoon (Tesla Model S) and evening (Nissan Leaf). In the case of RT Control (Fig. 5b) the Tesla Model S starts charging at 10:00 because it needs the energy for a trip, while the Nissan Leaf starts charging at 11:00 because then there is excess PV. Both EVs also charge when returned from the trip, since there is a small amount of excess PV. In the case of RT Control + V2G (Fig. 5c), both EVs start discharging in the night, because there is no PV to cover load demand. This results in a large peak in the morning for the Tesla Model S, since it does not have sufficient energy available for the trip during the day. The trip with the Nissan Leaf takes place later in the day, and because energy was discharged in the night more energy can be stored during the afternoon, resulting in a higher self-consumption of PV power than with RT Control. In the evening the energy available in the EVs is discharged to cover load demand. In the case of LP - Perfect Information (Fig. 5d), both EVs charge much less energy in the morning than in the previous case, because the system takes into account the planned trips. The total amount of discharged power is just enough to have the storage amount available to cover maximum self-consumption in the morning. In the evening the energy 


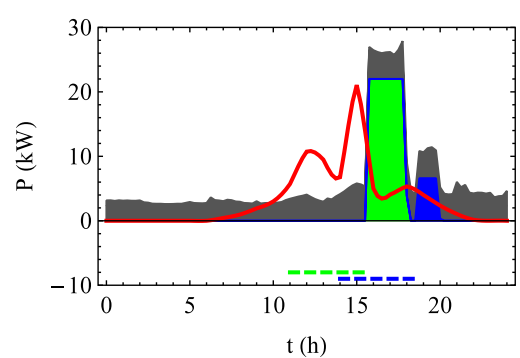

(a) No Control

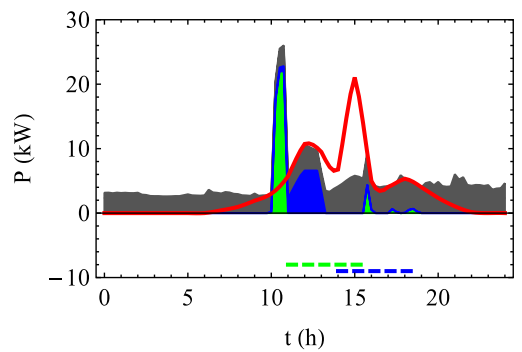

(b) RT Control

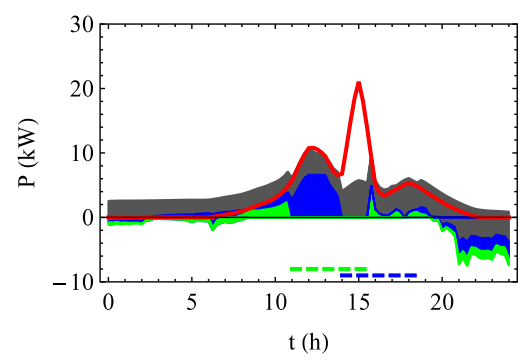

(d) LP - Perfect Information
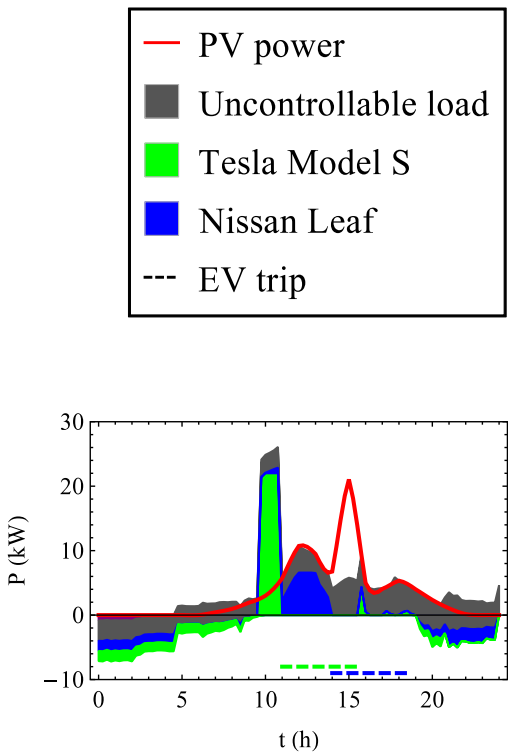

(c) RT Control + V2G

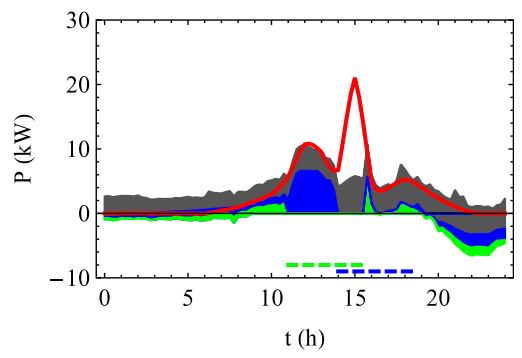

(e) LP - Uncertainties

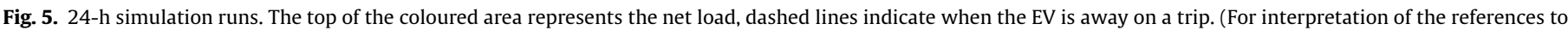
colour in this figure legend, the reader is referred to the web version of this article.)

available in the EVs is discharged to cover load demand. Note that the LP - Uncertainties (Fig. 5e) does not deviate much from LP Perfect Information, although the load curve is somewhat less flat due to deviations from the predictions.

\subsection{Simulation results}

Fig. 6 presents the load duration curves resulting from the simulations. The load curves clearly illustrate the reduced energy demand, energy sent to the grid and peak demand due to the control algorithms both at the demand and supply side. Peak demand due to charging of EVs for No Control is easily recognizable and indicated in the figure. Linear programming is better at reducing peaks in energy demand than the other charging strategies. Furthermore, the difference between the curve of LP - Perfect Information and LP - Uncertainties is small and is be visible only at the negative side (excess PV) of the graph.

Table 2 presents the indicator scores for each algorithm. Based on our sensitivity analysis on the effect of using 24-h simulations instead of month simulations, we lowered all indicator scores of LP with $7 \%$.

All proposed control systems contribute significantly to increasing self-consumption, reducing the energy sent to the grid and reducing peaks in electricity demand. The linear programming algorithms score highest on all indicators, also when uncertainties are taken into account. The advantage of V2G is also clear from the results, since scores on all indicators are higher for the algorithms that include V2G.

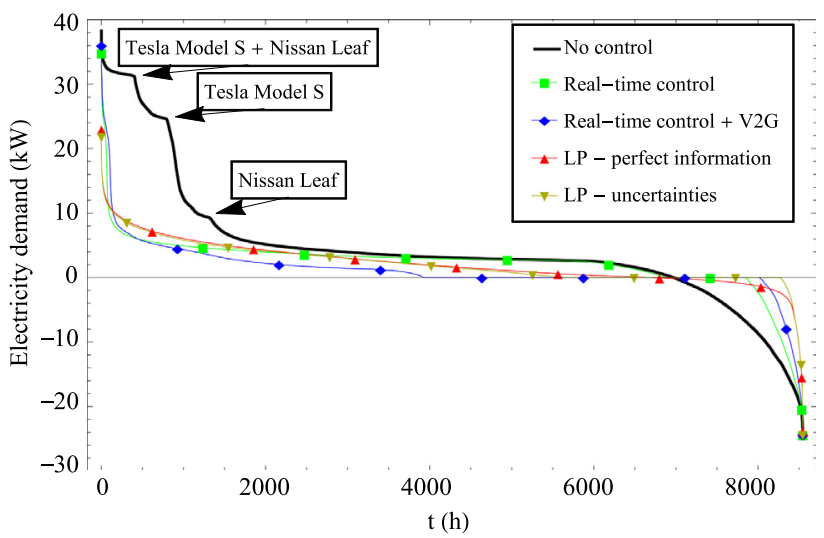

Fig. 6. Load duration curves resulting from simulations.

Table 2

Simulation results for system performance indicators.

\begin{tabular}{llll}
\hline Algorithm & SC (\%) & Energy to grid (MW h/yr) & RPR (\%) \\
\hline No Control & 49 & 12.4 & - \\
RT Control & 62 & 9.1 & 27 \\
RT Control + V2G & 79 & 4.8 & 43 \\
LP - Perfect Information & 91 & 2.0 & 75 \\
LP - Uncertainties & 87 & 3.4 & 67 \\
\hline
\end{tabular}




\subsection{Battery degradation}

Table 3 and Fig. 7 show our results for battery degradation indicators. The yearly energy throughput is the same for No Control and RT Control, since in both cases energy is only discharged during EV trips. For RT Control + V2G the energy throughput for the Tesla Model S is increased by factor 2.3 and for the Nissan Leaf by factor 4.0. Compared to using no V2G, using LP increases energy throughput by factor 3.0 for the Tesla Model $S$ and by factor 4.0 for the Nissan Leaf. The V2G option thus dramatically increases the use of the battery.

In the case of No Control, the EVs have an SOC below $100 \%$ only when charging. Therefore, the average SOC is very high (99\%). The average SOC resulting from the control algorithms is much lower, which will have a positive effect on battery lifetime.

In the case of No Control, the EVs always charge at maximum capacity. The average charging power resulting from the control algorithms is considerably lower, which will have a positive effect on battery lifetime. Fig. 7 shows the frequency distributions of charging power. The results show that discharging occurs only at relatively low charging rates. Furthermore, charging at maximum capacity occurs most often for RT Control, $11 \%$ of the total charging time for the Tesla Model S and 28\% of the total charging time for the Nissan Leaf, and barely $(<2 \%)$ for LP.

Summarising, using RT Control will result in minimum battery degradation, since the charging rates and average SOC are much lower than for No Control. V2G will have a significant impact on battery lifetime due to larger energy throughput, which was identified in the literature as the strongest indicator for battery degradation due to V2G. Of the two V2G options, LP is favourable, since it has the lowest charging rates.

Table 3

Simulation results for battery degradation indicators.

\begin{tabular}{|c|c|c|c|c|c|c|}
\hline \multirow[t]{2}{*}{ Algorithm } & \multicolumn{2}{|c|}{$\begin{array}{l}\text { Energy } \\
\text { throughput } \\
\text { (MW h/yr) }\end{array}$} & \multicolumn{2}{|c|}{$\mathrm{SOC}_{a v g}(\%)$} & \multicolumn{2}{|c|}{$P_{\text {avg }}(\mathrm{kW})$} \\
\hline & Model S & Leaf & Model S & Leaf & Model S & Leaf \\
\hline No Control & 6.9 & 2.4 & 99 & 99 & 22 & 6.6 \\
\hline RT Control & 6.9 & 2.4 & 65 & 67 & 5.7 & 2.8 \\
\hline RT Control + V2G & 16 & 9.6 & 50 & 40 & 2.6 & 2.1 \\
\hline LP & 21 & 9.6 & 51 & 55 & 2.3 & 1.1 \\
\hline
\end{tabular}

\subsection{Changes in microgrid composition}

LomboXnet is considering to expand the microgrid, see Section 2.4. We have performed simulations for these expansions; results are presented in Fig. 8. Our results show that upscaling will lead to lower self-consumption and peak reduction if V2G is used. In the absence of V2G, self-consumption and peak reduction is slightly higher in the upscaled microgrid, although in absolute terms energy sent to the grid increases.

Fig. 9 presents our results for variations in trips per week. These results imply that system performance is higher when the EVs make few trips: their function as electricity storage is more important than their function as flexible demand source. Only without a control system making more trips per week increases self-consumption, although only slightly because most of the time the EVs are charged when PV power production is low.

Fig. 10 presents our results for variation of EV type. The results clearly show that for the microgrid a Tesla Model S, with greater battery capacity and charging power, leads to better system performance than a Nissan Leaf.

\section{Discussion}

In this study we show the increase in self-consumption due to both V2G and optimisation using predictions. Our LP algorithm mainly serves as a way to illustrate the benefits of an optimisation algorithm over the RT algorithms for smart charging. Factors not incorporated in the objective function could be of interest to users of the technology, such as costs or power quality. Furthermore, the algorithm can be improved by enabling dynamic updating, which would allow it to respond to deviations from predictions during the day.

For the LP control algorithm we have simulated uncertainties in PV power and load demand predictions. However, uncertainties in EV trip times and energy use may arise as well. In our case, these uncertainties will arise for the return time of the EVs, since the EVs will often not have sufficiently charged before the planned starting time of the trip. If the EVs will return earlier then planned, this will have no effect on the charging pattern. If the EVs return later then planned, this will have an effect, because the EV is not available at the charging station. This will not occur often at LomboXnet, since trip times are agreed upon before the cars are rented out. However, to give an indication of how large this effect

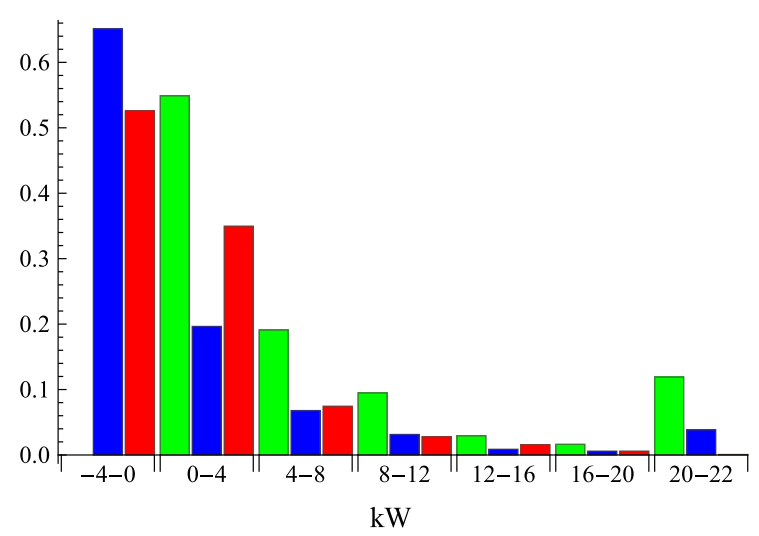

(a) Charging power Tesla Model S

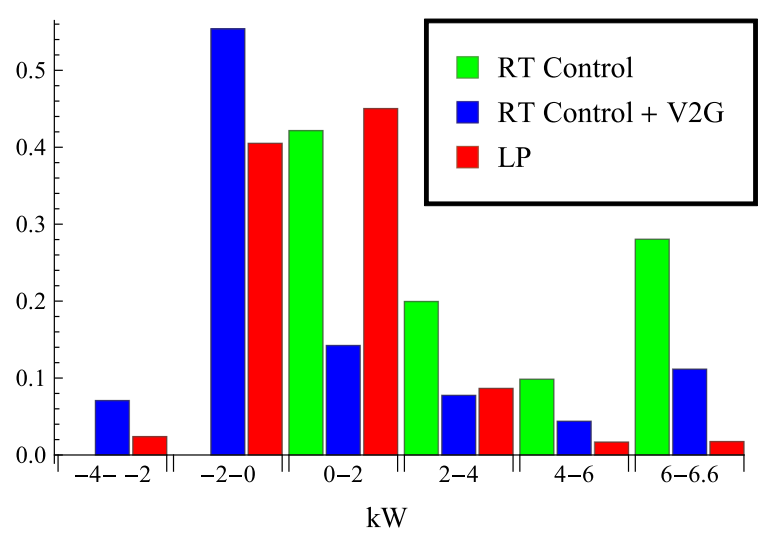

(b) Charging power Nissan Leaf

Fig. 7. Frequency distributions for charging power 


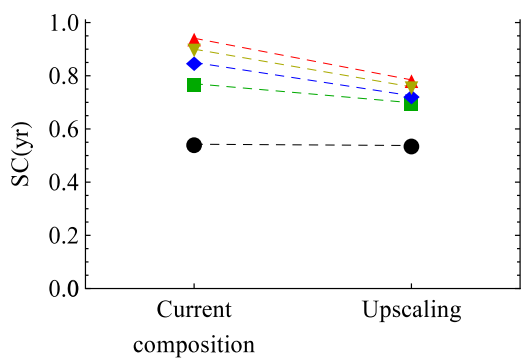

$$
\begin{aligned}
& \text { - No Control } \\
& \text { - RT Control } \\
& \text { - } \text { RT Control + V2G } \\
& \text { - LP - Perfect Information } \\
& \checkmark \text { LP - Uncertainties }
\end{aligned}
$$

(a) Self-consumption

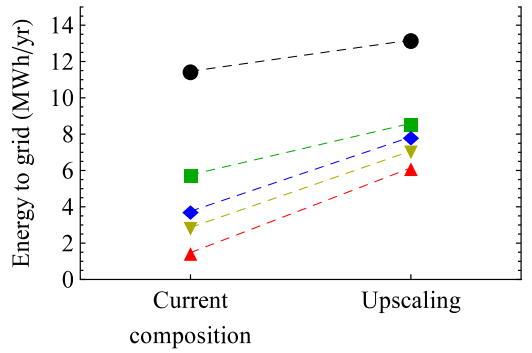

(b) Energy to grid

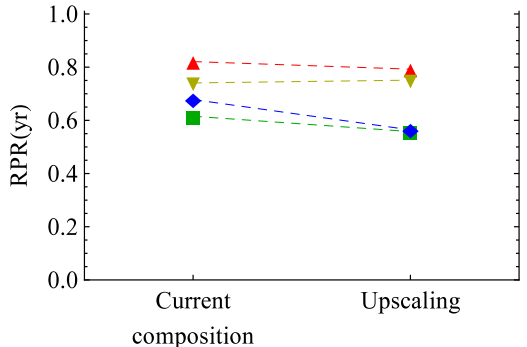

(c) Relative peak reduction

Fig. 8. Results for upscaling of microgrid.

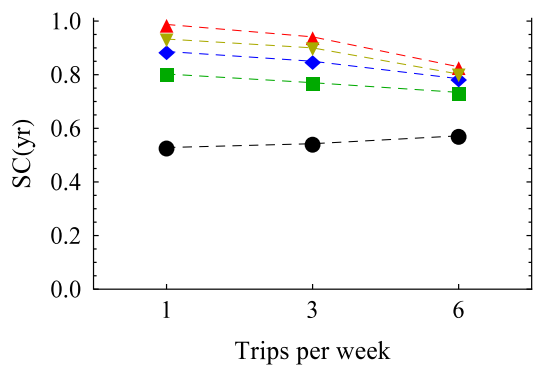

$$
\begin{aligned}
& \text { - No Control } \\
& \text { - } \text { RT Control } \\
& \text { - } \text { RT Control + V2G } \\
& \text { - LP - Perfect Information } \\
& \text { - LP - Uncertainties }
\end{aligned}
$$

(a) Self-consumption

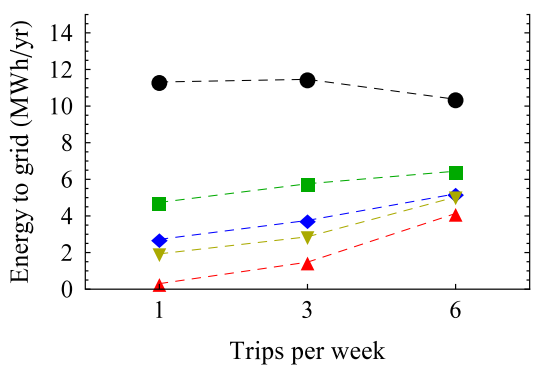

(b) Energy to grid

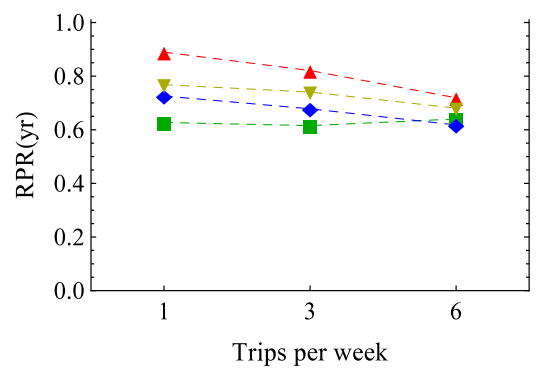

(c) Relative peak reduction

Fig. 9. Results for variations in EV trips.

can be, we have quantified the effect on self-consumption in the extreme case that the EVs are always returned one hour later then planned. We found that self-consumption is decreased with $2 \%$, indicating that uncertainties in trips will have a negligible effect on system performance. However, it should be noted that when such an optimisation algorithm is to be implemented in practice it should incorporate updating every time an EV is returned to be able to deal with these uncertainties.

Based on our indicator set, it is not possible to quantify the impact of the different control algorithms on battery degradation. To use a complete battery model, more information is needed on operating conditions, such as temperature and voltage. Two studies $[36,43]$ that quantified the impact of V2G on battery lifetime stated that energy throughput is the strongest indicator of battery degradation, but have found different relationships of energy throughput and battery degradation. Furthermore, it is not clear how battery degradation due to charging at the charging station compares to battery degradation due to driving and calendric lifetime. However, we can make some qualitative statements on this issue. In a more complete simulation model one of the several available battery models can be used to quantify the impacts on battery lifetime. 


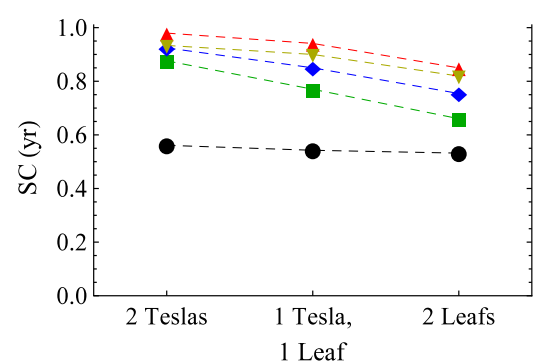

(a) Self-consumption

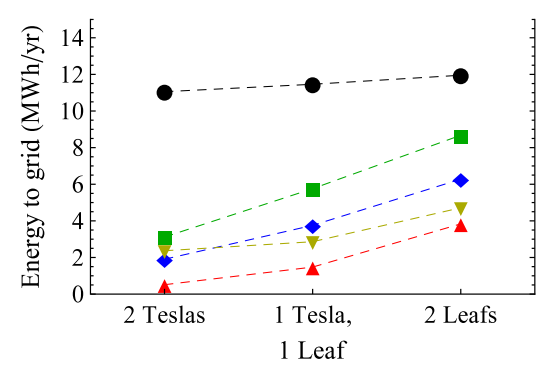

(b) Energy to grid
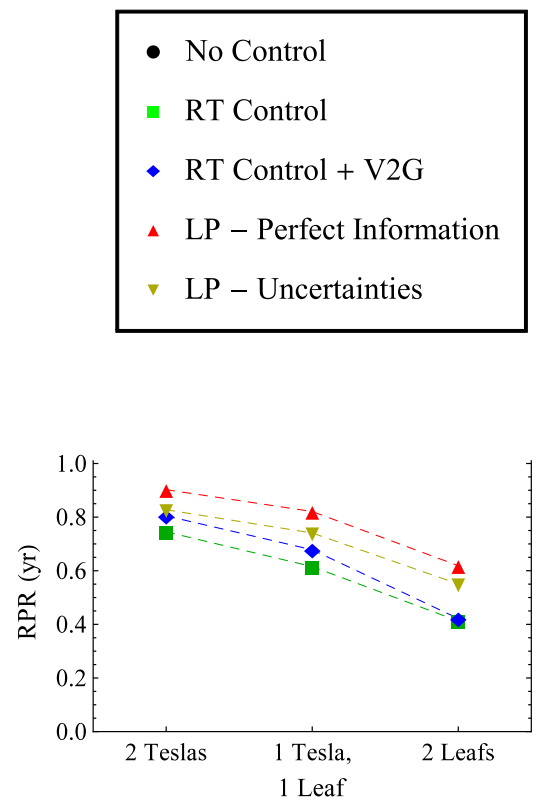

(c) Relative peak reduction

Fig. 10. Results for variations in EV type.

In our simulation we have used a minimum SOC of $20 \%$ to prevent overdischarge, similar as in [14]. However, several studies use a minimum SOC of $30 \%[16,34,44]$. We have performed additional simulations with a minimum SOC of $30 \%$. We found that using this higher minimum SOC has a small but significant impact on self-consumption, which is decreased with $1.5-2.0 \%$ for the control algorithms.

Our model contains some weaknesses that affect the quality of our results. Energy loss due to transport through the microgrid was not taken into account in the model. In a similar research, Claessen et al. [29] concluded that transport losses in a microgrid are significant. Furthermore, the decrease in maximum charging power of the batteries when nearly full has not been included in the model. The profile for the electricity demand of households is based on a dataset of 400 households from 2007 and 2008. Both measurements were taken at different locations at different times, so it is not known how closely they resemble a typical load demand pattern in Lombok. Moreover, the dataset contains information on aggregated load demand for one week in February; scaling the dataset for a year might not necessarily reflect how electricity demand changes throughout a year. Results can be improved by using a data-set measured in Lombok for a longer time period, preferably for a whole year. Finally, we were limited to using 24-h simulations for LP. If longer simulation times for LP can be used, this will give more accurate results.

\section{Conclusion}

In this paper we present a model developed to study the increase in self-consumption of PV power by smart charging EVs using smart grid technology. We apply this model to a case study: the microgrid of LomboXnet. We propose three EV charging control algorithms and have simulated their effect on self-consumption and peak reduction. The simulation results demonstrate that EVs can contribute significantly to well-balanced demand and supply. Self-consumption is increased from $49 \%$ to between $62 \%$ and $87 \%$, energy sent to the grid reduced from $12.4 \mathrm{MW} \mathrm{h}$ (26\% of total energy demand) to between 9.1 (19\%) and 3.4 (7\%) MW h and scores for relative peak reduction range from 0.27 to 0.67 . Our LP algorithm not only scores better for self-consumption than RT Control + V2G, but it also halves the largest peak in demand compared to the real-time algorithms, even when taking uncertainties in predictions into account.

The RT control algorithm has the lowest impact on battery lifetime, since not more energy is charged than needed for the EV trips and the charging rate and average SOC is lower than in the reference scenario of No Control. Using V2G dramatically increases battery use and will have a significant impact on battery lifetime. The benefits of $\mathrm{V} 2 \mathrm{G}$ will have to be weighed against this issue. Of the two V2G algorithms, LP will have the smallest impact on battery lifetime, since it has the lowest charging rates. In further research at LomboXnet we plan to empirically quantify the impact of V2G on battery lifetime.

We have shown the effect of changes in microgrid composition. Upscaling the microgrid will lower the scores for our indicators. Furthermore, the results for variations in trips per week show that our smart grid works best for situations where the EVs are regularly situated at the charging station. However, even when EVs make six trips per week, self-consumption will improve when using smart charging and V2G. Finally, our results indicate that using a Tesla Model S (or similar) is preferable to using a Nissan Leaf (or similar), due to the larger battery capacity and charging power of the first.

Despite several model limitations, our results clearly demonstrate the benefits of using smart charging and V2G in a microgrid and show how different sustainable energy and transport technologies can be combined in a manner that will reduce any negative impact on the existing energy infrastructure.

\section{Acknowledgments}

The authors would like to thank Robin Berg and Floris Bruning from LomboXnet for their input and providing the data used for the PV installations and their office demand, Felix Claessen for providing the electricity demand data for households, and Floortje Alkemade and two anonymous referees for helpful comments on the manuscript. 


\section{References}

[1] Elsinga B, Van Sark W. Spatial power fluctuation correlations in urban rooftop photovoltaic systems. Prog Photovolt: Res Appl. 2014.

[2] Reich NH, Mueller B, Armbruster A, Sark WG, Kiefer K, Reise C. Performance ratio revisited: is $\mathrm{PR}>90 \%$ realistic? Prog Photovolt: Res Appl 2012;20(6):717-26.

[3] Castillo-Cagigal M, Caamaño-Marte E, Matallanas E, Masa-Bote D, Gutiérrez A, Monasterio-Huelin F, et al. PV self-consumption optimization with storage and active DSM for the residential sector. Solar Energy 2011;85(9):2338-48.

[4] Eising JW, van Onna T, Alkemade F. Towards smart grids: identifying the risks that arise from the integration of energy and transport supply chains. Appl Energy 2014;123:448-55.

[5] EPIA policy and communications working group. Self consumption of PV electricity, position paper. European Photovoltaic Industry Association, Renewable Energy House; July 2013.

[6] Verbong GP, Beemsterboer S, Sengers F. Smart grids or smart users? Involving users in developing a low carbon electricity economy. Energy Policy 2013;52:117-25.

[7] Matallanas E, Castillo-Cagigal M, Gutiérrez A, Monasterio-Huelin F, CaamañoMart $́$ E, Masa D, et al. Neural network controller for active demand-side management with PV energy in the residential sector. Appl Energy 2012;91(1):90-7.

[8] Foley A, Tyther B, Calnan P, Ó Gallachóir B. Impacts of electric vehicle charging under electricity market operations. Appl Energy 2013;101:93-102.

[9] Kempton W, Tomić J. Vehicle-to-grid power implementation: from stabilizing the grid to supporting large-scale renewable energy. J Power Sources 2005;144(1):280-94.

[10] Lund H, Kempton W. Integration of renewable energy into the transport and electricity sectors through V2G. Energy Policy 2008;36(9):3578-87.

[11] Andersson S-L, Elofsson A, Galus M, Göransson L, Karlsson S, Johnsson F, et al. Plug-in hybrid electric vehicles as regulating power providers: case studies of Sweden and Germany. Energy Policy 2010;38(6):2751-62.

[12] Hein R, Kleindorfer PR, Spinler S. Valuation of electric vehicle batteries in vehicle-to-grid and battery-to-grid systems. Technol Forecast Soc Change 2012;79(9):1654-71.

[13] Sousa T, Morais H, Soares J, Vale Z. Day-ahead resource scheduling in smart grids considering vehicle-to-grid and network constraints. Appl Energy 2012;96:183-93.

[14] Tulpule PJ, Marano V, Yurkovich S, Rizzoni G. Economic and environmental impacts of a PV powered workplace parking garage charging station. Appl Energy 2013;108:323-32.

[15] Birnie DP. Solar-to-vehicle (S2V) systems for powering commuters of the future. J Power Sources 2009;186(2):539-42.

[16] Zhang Q, Tezuka T, Ishihara KN, Mclellan BC. Integration of PV power into future low-carbon smart electricity systems with EV and HP in Kansai Area, Japan. Renew Energy 2012;44:99-108.

[17] Drude L, Pereira Jr LC, Rüther R. Photovoltaics (PV) and electric vehicle-to-grid (V2G) strategies for peak demand reduction in urban regions in Brazil in a smart grid environment. Renew Energy 2014;68:443-51.

[18] Tuffner F, Chassin F, Kintner-Meyer M, Gowri K. Utilizing electric vehicles to assist integration of large penetrations of distributed photovoltaic generation. Pacific Northwest National Laboratory; 2012.

[19] Guille C, Gross G. A conceptual framework for the vehicle-to-grid (V2G) implementation. Energy Policy 2009;37(11):4379-90.

[20] Sovacool BK, Hirsh RF. Beyond batteries: an examination of the benefits and barriers to plug-in hybrid electric vehicles (PHEVs) and a vehicle-to-grid (V2G) transition. Energy Policy 2009;37(3):1095-103.

[21] Kemp R, Schot J, Hoogma R. Regime shifts to sustainability through processes of niche formation: the approach of strategic niche management. Technol Anal Strat Manage 1998;10(2):175-98.

[22] Raven R. Niche accumulation and hybridisation strategies in transition processes towards a sustainable energy system: an assessment of differences and pitfalls. Energy Policy 2007;35(4):2390-400.
[23] Schot J, Geels FW. Strategic niche management and sustainable innovation journeys: theory, findings, research agenda, and policy. Technol Anal Strat Manage 2008;20(5):537-54.

[24] Shaheen SA, Cohen AP. Carsharing and personal vehicle services: worldwide market developments and emerging trends. Int J Sustain Transport 2013;7(1):5-34.

[25] Meijkamp R. Changing consumer behaviour through eco-efficient services: an empirical study of car sharing in the Netherlands. Business Strat Environ $1998 ; 7(4): 234-44$

[26] Martin EW, Shaheen SA. Greenhouse gas emission impacts of carsharing in North America. IEEE Trans Intell Transport Syst 2011;12(4):1074-86.

[27] Mont O, Neuvonen A, Lähteenoja S. Sustainable lifestyles 2050: stakeholder visions, emerging practices and future research. J Clean Prod 2014;63:24-32.

[28] Moraitis P, van Sark WGJHM. Operational performance of grid-connected PV systems. In: Conference record of the 40th IEEE photovoltaic specialists conference. Denver, CO, USA: IEEE; 2014. p. 1953-6.

[29] Claessen F, Claessens B, Hommelberg M, Molderink A, Bakker V, Toersche $H$, et al. Comparative analysis of tertiary control systems for smart grids using the Flex Street model. Renew Energy 2014;69:260-70.

[30] Dril Av, Gerdes J, Marbus S, Boelhouwer M. Energie trends 2012. Policy studies; 2012.

[31] González Vayá M, Andersson G. Centralized and decentralized approaches to smart charging of plug-in vehicles. In: Power and energy society general meeting. IEEE; 2012. p. 1-8.

[32] Guo Y, Pan M, Fang Y. Optimal power management of residential customers in the smart grid. IEEE Trans Paral Distrib Syst 2012;23(9):1593-606.

[33] Tanaka K, Yoza A, Ogimi K, Yona A, Senjyu T, Funabashi T, et al. Optimal operation of DC smart house system by controllable loads based on smart grid topology. Renew Energy 2012;39(1):132-9.

[34] Amirioun MH, Kazemi A. A new model based on optimal scheduling of combined energy exchange modes for aggregation of electric vehicles in a residential complex. Energy 2014;69:186-98.

[35] van der Kam MJ, van Sark WGJHM. Increasing self-consumption of photovoltaic electricity by storing energy in electric vehicle using smart grid technology in the residential sector. In: Proceedings of the 3rd international conference on smart grids and green IT systems, SMARTGREENS; 2014. p. 1420.

[36] Bishop JD, Axon CJ, Bonilla D, Tran M, Banister D, McCulloch MD. Evaluating the impact of V2G services on the degradation of batteries in PHEV and EV. Appl Energy 2013;111:206-18.

[37] Kempton W, Tomić J. Vehicle-to-grid power fundamentals: calculating capacity and net revenue. J Power Sources 2005;144(1):268-79.

[38] Peterson SB, Whitacre J, Apt J. The economics of using plug-in hybrid electric vehicle battery packs for grid storage. J Power Sources 2010;195(8):2377-84.

[39] Marano V, Onori S, Guezennec Y, Rizzoni G, Madella N. Lithium-ion batteries life estimation for plug-in hybrid electric vehicles. In: Vehicle power and propulsion conference, 2009. VPPC'09. IEEE. IEEE; 2009. p. 536-43.

[40] Lunz B, Yan Z, Gerschler JB, Sauer DU. Influence of plug-in hybrid electric vehicle charging strategies on charging and battery degradation costs. Energy Policy 2012;46:511-9.

[41] Lam L, Bauer P, Kelder E. A practical circuit-based model for Li-ion battery cells in electric vehicle applications. In: 2011 IEEE 33rd international, telecommunications energy conference (INTELEC). IEEE; 2011. p. 1-9.

[42] Hoke A, Brissette A, Maksimovic D, Pratt A, Smith K. Electric vehicle charge optimization including effects of lithium-ion battery degradation. In: 2011 IEEE vehicle power and propulsion conference (VPPC). IEEE; 2011. p. 1-8.

[43] Peterson SB, Apt J, Whitacre J. Lithium-ion battery cell degradation resulting from realistic vehicle and vehicle-to-grid utilization. J Power Sources 2010;195(8):2385-92.

[44] Khayyam H, Ranjbarzadeh H, Marano V. Intelligent control of vehicle to grid power. J Power Sources 2012;201:1-9. 